\title{
Exosome-Mediated Activation of Neuronal Cells Triggered by $\gamma$-Aminobutyric Acid (GABA)
}

\author{
Ryo Inotsuka ${ }^{1}$, Miyako Udono ${ }^{2}$, Atsushi Yamatsu ${ }^{3,4}$, Mujo Kim ${ }^{4}$ and Yoshinori Katakura ${ }^{1,2, *(D)}$ \\ 1 Graduate School of Bioresources and Bioenvironmental Sciences, Kyushu University, Fukuoka 819-0395, \\ Japan; inotsuka.ryo.840@s.kyushu-u.ac.jp \\ 2 Faculty of Agriculture, Kyushu University, Fukuoka 819-0395, Japan; mudono@grt.kyushu-u.ac.jp \\ 3 International GABA Research Center, Kyoto 615-8245, Japan; a-yamatsu@pharmafoods.co.jp \\ 4 Pharma Foods International Co., Ltd., Kyoto 615-8245, Japan; mujokim@pharmafoods.co.jp \\ * Correspondence: katakura@grt.kyushu-u.ac.jp
}

check for updates

Citation: Inotsuka, R.; Udono, M.; Yamatsu, A.; Kim, M.; Katakura, Y. Exosome-Mediated Activation of Neuronal Cells Triggered by $\gamma$-Aminobutyric Acid (GABA) Nutrients 2021, 13, 2544.

https://doi.org/10.3390/nu13082544

Academic Editor: Paolo Brambilla

Received: 1 June 2021

Accepted: 23 July 2021

Published: 25 July 2021

Publisher's Note: MDPI stays neutral with regard to jurisdictional claims in published maps and institutional affiliations.

Copyright: (c) 2021 by the authors. Licensee MDPI, Basel, Switzerland. This article is an open access article distributed under the terms and conditions of the Creative Commons Attribution (CC BY) license (https:/ / creativecommons.org/licenses/by/ $4.0 /)$.

\begin{abstract}
Aminobutyric acid (GABA) is a potent bioactive amino acid, and several studies have shown that oral administration of GABA induces relaxation, improves sleep, and reduces psychological stress and fatigue. In a recent study, we reported that exosomes derived from GABA-treated intestinal cells serve as signal transducers that mediate brain-gut interactions. Therefore, the purpose of this study was to verify the functionality of GABA-derived exosomes and to examine the possibility of improving memory function following GABA administration. The results showed that exosomes derived from GABA-treated intestinal cells (Caco-2) activated neuronal cells (SH-SY5Y) by regulating genes related to neuronal cell functions. Furthermore, we found that exosomes derived from the serum of GABA-treated mice also activated SH-SY5Y cells, indicating that exosomes, which are capable of activating neuronal cells, circulate in the blood of mice orally administered GABA. Finally, we performed a microarray analysis of mRNA isolated from the hippocampus of mice that were orally administered GABA. The results revealed changes in the expression of genes related to brain function. Gene Set Enrichment Analysis (GSEA) showed that oral administration of GABA affected the expression of genes related to memory function in the hippocampus.
\end{abstract}

Keywords: GABA; exosome; gut-brain interaction; Caco-2; SH-SY5Y

\section{Introduction}

$\gamma$-Aminobutyric acid (GABA) is a naturally occurring nonprotein amino acid that is one of the principal inhibitory neurotransmitters of the central nervous system (CNS) [1]. The human $\mathrm{GABA}_{\mathrm{B}}$ receptor-a member of the class $\mathrm{C}$ family of G-protein-coupled receptors (GPCRs)-mediates inhibitory neurotransmission [2]. It has been reported that GABAergic synapses constitute $20-50 \%$ of all synapses present in the CNS [3] and play important roles in information encoding and behavioral control, regulation of motor learning, and motor functions $[4,5]$. Generally, genes characterized by GABAergic function are down-regulated during normal aging in humans [6], and in certain studies involving animals, it has been suggested that GABA function declines with age. For example, the number of GABAimmunoreactive neurons declines with age in the hippocampus, inferior colliculus, and striate visual cortex of animals [7-9]. There are also reported reductions in GABA levels, GABA release, and GABA receptor binding from the baseline levels with aging [8]. When the concentration of GABA in the brain diminishes below a certain threshold, various neurological disorders including epilepsy, seizures, convulsions, and Alzheimer disease have also been reported [10-12]. Furthermore, it has been recently reported that serum GABA levels are decreased in various diseases including stroke [13]. These studies suggest that GABA has health-promoting effects that may also be related to the enhancement of brain function. 
The development of functional foods containing GABA has been actively studied. In a recent study, a randomized, double-blind, placebo-controlled clinical trial involving healthy Japanese adults was reported, where administration of $100 \mathrm{mg}$ of GABA resulted in increased participant memory and spatial cognitive function [14]. Indeed, several studies have shown that oral administration of GABA induces relaxation, improves sleep, and reduces psychological stress and fatigue [15-17]. Additionally, healthy adults who consumed $50 \mathrm{mg}$ of GABA dissolved in a beverage reported less occupational fatigue after completion of required tasks [18]. However, in view of the lack of evidence regarding the blood-brain barrier permeability of GABA, the mechanisms through which GABA might exert these beneficial effects in humans remain unclear. It is considered that the oral intake of GABA exerts such effects through an indirect pathway [19].

Furthermore, GABA has been reported to have a variety of health promoting effects including immunomodulatory, anti-diabetes, anti-cancer, anti-oxidant, and so on [20-23]. Future research is expected to focus not only on neurological and psychological disorders linked to a decrease in amount of GABA, but also on the systemic health-promoting effects of GABA and its molecular basis.

In our previous study, we clarified the molecular basis of GABA-induced gut-brain interactions and reported that exosomes derived from GABA-treated intestinal cells serve as signal transducers that mediate brain-gut interactions [24]. Exosomes are a family of particles released from the cell that are delimited by a lipid bilayer, and attention has recently been focused on the role of exosomes as biomarker candidates for diagnosis, prognosis and even therapeutic tools of various diseases [25]. In the present study, we clarified that exosomes derived from serum of mice administered GABA as well as from GABA-treated Caco-2 cells activated neuronal cells, and that GABA administration changes the expression of memory-related genes in hippocampus.

\section{Materials and Methods}

\subsection{Cell Culture and Reagents}

The human colorectal cancer cell line Caco-2 (ATCC, Manassas, VA, USA) and the human neuroblastoma cell line SH-SY5Y (ATCC) were cultured in Dulbecco's modified Eagle's medium (DMEM; Nissui, Tokyo, Japan) supplemented with 10\% heat-inactivated fetal bovine serum (FBS, Life Technologies, Gaithersburg, MD, USA) at $37^{\circ} \mathrm{C}$ in an atmosphere containing $5 \% \mathrm{CO}_{2} . \gamma$-Aminobutyric acid (GABA) was purchased from Abcam (Cambridge, UK). 5-Aminoimidazole-4-carboxamide 1- $\beta$-D-ribofuranoside (AICAR) and retinoic acid (RA) were purchased from FUJIFILM Wako Pure Chemical Corp. (Osaka, Japan).

\subsection{Exosome Isolation and Treatment}

Firstly, Caco- 2 cells $\left(1.4 \times 10^{5}\right.$ cells $\left./ \mathrm{mL}\right)$ were cultured in DMEM containing $10 \%$ Exosome-depleted FBS media supplement heat inactivated (System Bioscience, Mountain View, CA, USA) and 500 or $1000 \mu \mathrm{M}$ GABA. After $24 \mathrm{~h}$ of culture, the MagCapture Exosome Isolation Kit PS (FUJIFILM Wako Pure Chemical Corp.) was used to isolate exosomes from the media of Caco-2 cells, according to the manufacturer's instructions. Exosomes were isolated from mouse serum using ExoQuick Exosome Precipitation Solution (System Biosciences, Palo Alto, CA, USA), according to the manufacturer's instructions. SH-SY5Y cells $\left(2.0 \times 10^{5}\right.$ cell $\left./ \mathrm{mL}\right)$ were cultured for $24 \mathrm{~h}$, and treated with exosomes (equivalent to $90 \mathrm{ng}$ protein) derived from GABA-treated Caco-2 cells for $24 \mathrm{~h}$.

\subsection{Quantitative Evaluation of Neurite Growth}

SH-SY5Y cells were seeded onto a $\mu$ Clear fluorescence black plate (Greiner-Bio One, Tokyo, Japan), fixed with $4 \%$ paraformaldehyde for $15 \mathrm{~min}$, and blocked with blocking buffer $(1 \times$ PBS, $5 \%$ goat serum, and $0.3 \%$ Triton X-100) for $1 \mathrm{~h}$. The cells were subsequently incubated with Milli-Mark Pan Neuronal Marker (Merck Millipore, Billerica, MA, USA) at $25^{\circ} \mathrm{C}$ overnight. After washing with PBS, the cells were stained with Alexa Fluor 555 goat 
anti-rabbit IgG antibody (Thermo Fisher Scientific, Inc., Waltham, MA, USA) for $1 \mathrm{~h}$ at $25^{\circ} \mathrm{C}$. After washing with PBS, cells were further stained with Hoechst 33342 (Dojindo, Kumamoto, Japan) for $15 \mathrm{~min}$, and neurite length was measured using the IN Cell Analyzer 2200 (GE Healthcare, Amersham Place, UK), as previously described [24]. The total neurite length for each cell is shown in the figure.

\subsection{Mitochondria}

Cells were stained with $250 \mathrm{nM}$ MitoTracker Red CMXRos (Thermo Fischer Scientific) at $37^{\circ} \mathrm{C}$ for $30 \mathrm{~min}$, and subsequently with $200 \mathrm{nM}$ MitoTracker Green FM (Thermo Fischer Scientific) at $37^{\circ} \mathrm{C}$ for $30 \mathrm{~min}$. Finally, the cells were stained with Hoechst 33342 at $37^{\circ} \mathrm{C}$ for $30 \mathrm{~min}$. Stained cells were analyzed using IN Cell Analyzer 2200 (GE Healthcare, Amersham Place, UK) to quantitatively determine the number, area, and activity of mitochondria using IN Cell Investigator high-content image analysis software (GE Healthcare).

\subsection{Quantitative Reverse Transcriptase-Polymerase Chain Reaction (RT-qPCR)}

RNA was prepared from cells using the High Pure RNA Isolation kit (Roche Diagnostics $\mathrm{GmbH}$, Mannheim, Germany) according to the manufacturer's protocols. RT-qPCR was performed using the GoTaq 1-Step RT-PCR System (Promega, WI, USA) and Thermal Cycler Dice Real Time System TP-800 (Takara). Samples were analyzed in triplicate. The PCR primer sequences used were as follows: human $\beta$-actin $(A C T B)$ forward primer $5^{\prime}$ TGGCACCCAGCACAATGAA- $3^{\prime}$ and reverse primer $5^{\prime}$-CTAAGTCATAGTCCGCCTAGAA GCA- $3^{\prime}$ : human brain-derived growth factor (BDNF) forward primer $5^{\prime}$-GTCAAGTTGGGA GCCTGAAATAGTG-3' and reverse primer $5^{\prime}$-AGGATGCTGGTCCAAGTGGTG-3': peroxisome proliferator-activated receptor $\gamma$ coactivator $1-\alpha(P G C-1 \alpha)$ forward primer $5^{\prime}$ GCTGACAGATGGAGACGTGA- $3^{\prime}$ and reverse primer $5^{\prime}$-TAGCTGAGTGTTGGCTGGTG$3^{\prime}$ : human NESTIN forward primer $5^{\prime}$-ACTGGGAAGGAGGAGGTGGT-3' and reverse primer 5'-CACACTGGCTCCCTCAACCA-3': human neurofilament medium chain (NEFM) forward primer $5^{\prime}$-AGACATCCACCGGCTCAAGG-3' and reverse primer $5^{\prime}$-CGACGCCTC CTCGATGTCT- $3^{\prime}$. $\beta$-actin was used as a housekeeping gene. Samples were normalized and analyzed by the $\Delta \Delta C T$ method [26].

\section{6. miRNA Microarray Assay}

The expression profile of miRNA in exosomes prepared using the MagCapure Exosome Isolation Kit PS was evaluated using microarray analysis with an Affimetrix GeneChip miRNA 4.0 Array (Affymetrix, Santa Clara, CA, USA). Total RNA was isolated from exosomes using TRIzol Reagent (Thermo Fisher Scientific) and purified using the miRNeasy Mini Kit (Qiagen, Valencia, CA, USA). Subsequent operations were outsourced to Cell Innovator (Fukuoka, Japan), a commercial contract analysis provider. To identify up- or down-regulated genes, we calculated ratios (non-log scaled fold-change) from the normalized intensities of each gene for comparisons between control and experimental samples. Then, we established criteria for regulated genes: (up-regulated genes) ratio $\geq$ 1.3-fold; (down-regulated genes) ratio $\leq 0.77$ [27]. miRNA target genes were predicted using miRWalk (http:/ / mirwalk.umm.uni-heidelberg.de, access on 20 February 2021). To determine significantly over-represented gene ontology (GO) categories and significantly enriched pathways, we used tools and data provided by the Database for Annotation, Visualization, and Integrated Discovery (DAVID, http:/ / david.abcc.ncifcrf.gov, access on 20 February 2021) [28,29].

\section{7. mRNA Microarray Assay}

The mRNA expression profile was evaluated using a DNA microarray (SurePrint G3 Human Gene Expression $8 \times 60 \mathrm{~K}$ v.3, Agilent). Total RNA was isolated from SH-SY5Y cells and mouse brains using Isogen II (Nippon Gene, Tokyo, Japan). The subsequent operations were outsourced to Cell Innovator. We then established criteria for significantly up- or down-regulated genes: up-regulated genes, Z-score $\geq 2.0$ and ratio $\geq 1$.5-fold; down- 
regulated genes: Z-score $\leq-2.0$ and ratio $\leq 0.66$-fold. Significantly over-represented GO categories and enriched pathways were determined as described above. Gene set enrichment analysis (GSEA) was performed to determine the enrichment score (ES), which indicates the degree to which each gene set is overrepresented at the top or bottom of a ranked list of genes.

\subsection{Integrated Analysis}

We then performed integrated analysis of miRNAs with altered expression in ExoGABA and of mRNAs with altered expression in SH-SY5Y cells in response to Exo-GABA treatment, and selected genes.

\subsection{Animal Experiments}

Six-week-old female C57BL/ 6 mice were obtained from KBT Oriental Co. Ltd. (Saga, Japan) and allowed to adapt for 2 weeks, with food and water provided ad libitum. All mouse experiments and protocols were in accordance with the Guide for the Care and Use of Laboratory Animals and were approved by the Ethics Committee on Animal Experimentation (Kyushu University; approval number: A28-187-0). One group was composed of five mice. GABA solution was orally administered to mice once a day in $100 \mu \mathrm{L}(200 \mathrm{mg} / \mathrm{kg})$ doses using a sonde. The control group received $100 \mu \mathrm{L}$ of sterile water orally. After 7 days of treatment, cardiac blood samples were collected from the mice, and serum samples were prepared. Serum was used for exosome isolation by using the MagCapture Exosome Isolation Kit PS. Simultaneously, hippocampal tissue was collected from mouse brains.

\subsection{Statistical Analysis}

All experiments were performed at least three times, and the corresponding data are shown. The results are presented as means \pm standard deviation. Multiple comparisons between groups were carried out by one-way ANOVA with Tukey's post-hoc test. Statistical significance was defined as $p<0.05\left({ }^{*} p<0.05 ;{ }^{* *} p<0.01 ;{ }^{* * *} p<0.001\right)$.

\section{Results}

\subsection{Exosomes Derived from GABA-Treated Caco-2 Cells Activate SH-SY5Y Cells}

Caco-2 cells are used in this study because they are known to exhibit differentiation functions such as digestion and absorption. First, we tested whether exosomes derived from GABA-treated Caco-2 cells activated SH-SY5Y cells. Exo-ctrl shows the exosomes derived from non-treated Caco-2 cells. As shown in Figure 1A, although the treatment of Caco-2 cells with 500 or $1000 \mu \mathrm{M}$ GABA did not change the amount of exosomes that could be isolated (data not shown), exosomes derived from GABA-treated Caco-2 cells (Exo-GABA) exhibited elongated neurites in SH-SY5Y cells as compared to Exoctrl. However, the neurite outgrowth effect of Exo-GABA was lower than that of the positive control retinoic acid (RA). Furthermore, Exo-GABA was found to augment the expression of $P G C-1 \alpha$, a master gene of mitochondrial biogenesis, and increased the number, area, and activity of mitochondria in SH-SY5Y cells (Figure 1B-E), as compared to Exoctrl. 5-Aminoimidazole-4-carboxamide 1- $\beta$-D-ribofuranoside (AICAR) is known to induce mitochondrial biogenesis [30], and then used as a positive control. As can be seen from some of the results, exosomes derived from Caco-2 cells treated with $1000 \mu \mathrm{M}$ GABA were found to be more effective. 
A

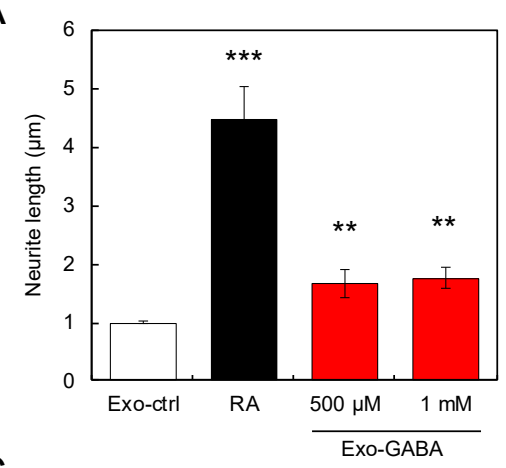

C

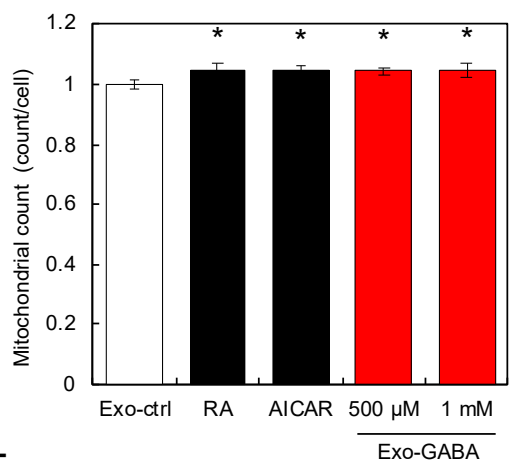

$E$

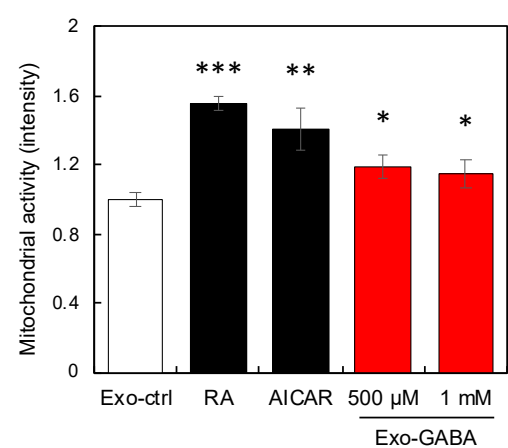

$\mathrm{B}$

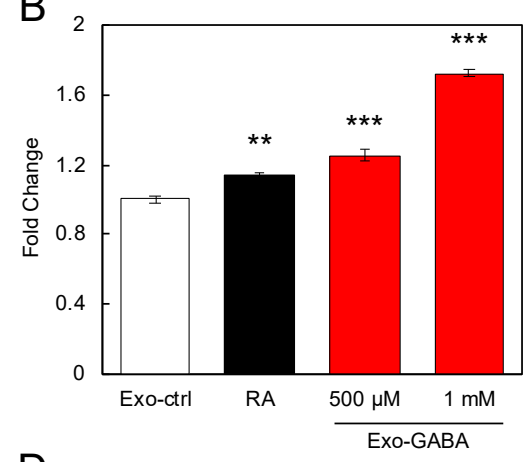

D

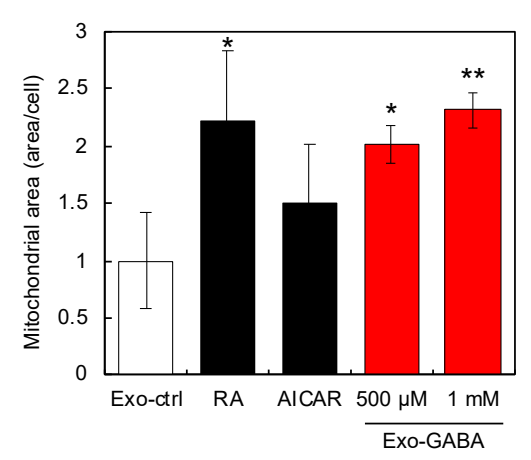

Figure 1. Exosomes derived from GABA-treated Caco-2 cells activate SH-SY5Y cells. Neurite length (A) and PGC-1 $\alpha$ expression (B) in SH-SY5Y cells treated with exosomes (equivalent to 90 ng protein) derived from GABA-treated Caco-2 cells. Exo-ctrl shows the exosomes derived from non-treated Caco-2 cells. Count (C), area (D), and activity (E) of mitochondria in SH-ST5Y cells treated with exosomes derived from GABA-treated Caco-2 cells. Retinoic acid (RA) was used as positive control. Multiple comparisons between groups were carried out by one-way ANOVA with Tukey's post-hoc test. Statistical difference was evaluated by comparing to Exo-ctrl. Statistical significance was defined as $p<0.05\left({ }^{*} p<0.05 ;{ }^{* *} p<0.01 ; * * * 0.001\right.$ ) (value means $\pm \mathrm{SEM}, n=3$ ).

Next, we tested the effects of Exo-GABA on the expression of marker genes (brainderived growth factor, $B D N F$; Nestin; and neurofilament medium chain, NEFM) of neuronal cells in SH-SY5Y cells. The results showed that Exo-GABA augmented the expression of these marker genes (Figure 2A-C). These results indicated that Exo-GABA activated SH-SY5Y cells. 
A
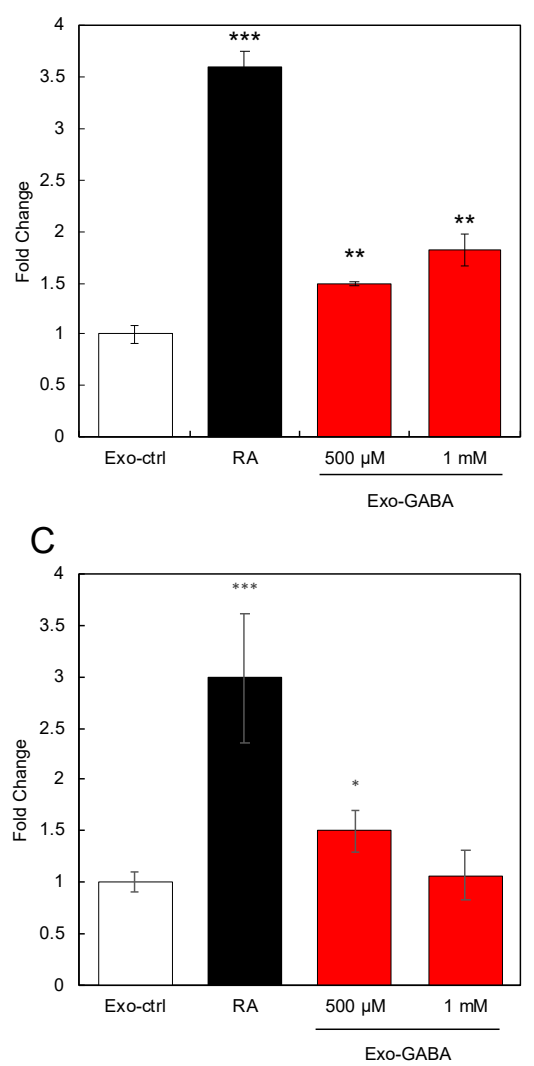

B

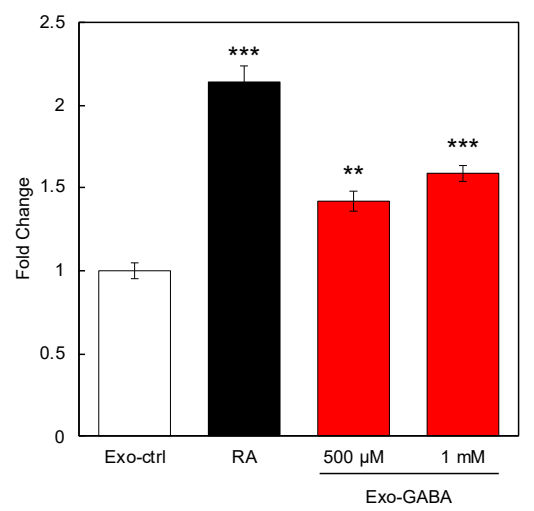

Figure 2. Effects of exosomes derived from GABA-treated Caco-2 cells on gene expression. Expression of brain-derived growth factor (BDNF) (A), Nestin (B), and neurofilament medium chain (NEFM) (C) in SH-SY5Y cells treated with exosomes derived from GABA-treated Caco-2 cells was evaluated by RT-qPCR. Retinoic acid (RA) was used as positive control. Multiple comparisons between groups were carried out by one-way ANOVA with Tukey's post-hoc test. Statistical difference was evaluated by comparing to Exo-ctrl. Statistical significance was defined as $p<0.05\left({ }^{*} p<0.05 ;{ }^{* *} p<0.01\right.$; *** $p<0.001$ ) (value means \pm SEM, $n=3$ ).

\subsection{Molecular Basis for the Exo-GABA-Induced Activation of SH-SY5Y}

We then tried to identify miRNAs contained in Exo-GABA and their target genes that activate SH-SY5Y cells using integrated analysis. Exo-ctrl, Exo-GABA ${ }_{500}$, and Exo$\mathrm{GABA}_{1000}$ show the exosomes derived from non-treated, $500 \mu \mathrm{M}$ GABA-treated and $1000 \mu \mathrm{M}$ GABA-treated Caco-2 cells, respectively. First, microarray analysis of miRNAs in Exo-GABA was used to search for miRNAs in exosomes that varied with GABA treatment. The results showed that GABA treatment decreased the expression levels of the four miRNAs in Caco-2 cells (Table 1). The target gene of these four miRNAs can be found in the Supplementary Materials Table S1. KEGG pathway analysis showed that all four miRNAs were involved in neuronal cell regulation and activation (Table 1).

Table 1. Functional classification of miRNAs differentially expressed in GABA-treated Caco-2 cells.

\begin{tabular}{ll}
\hline miRNA & KEGG Pathway \\
\hline miR-6732-5p & Calcium signaling pathway, Axon guidance, Neurotrophin signaling pathway \\
miR-8075 & $\begin{array}{l}\text { Calcium signaling pathway, Regulation of actin cytoskeleton, Long-term potentiation, Neurotrophin signaling } \\
\text { pathway, Axon guidance }\end{array}$ \\
miR-3665 & $\begin{array}{l}\text { Axon guidance, Long-term potentiation, Calcium signaling pathway, Neurotrophin signaling pathway } \\
\text { Axon guidance, Long-term potentiation, Calcium signaling pathway, Neurotrophin signaling pathway, Long-term } \\
\text { miR-5787 }\end{array}$ \\
\hline
\end{tabular}


Changes in mRNA expression in Exo-GABA-treated SH-SY5Y cells were examined using microarray analysis (Figure 3), and we identified 641 mRNAs whose expression was commonly altered in SH-SY5Y treated with Exo-GABA 500 and Exo-GABA 1000 . These genes can be directly or indirectly regulated by miRNAs and include both up-regulated and down-regulated genes. Similarly, using DAVID, KEGG pathway analysis estimated the involvement of pathways implicated in neuronal activity (Table 2).

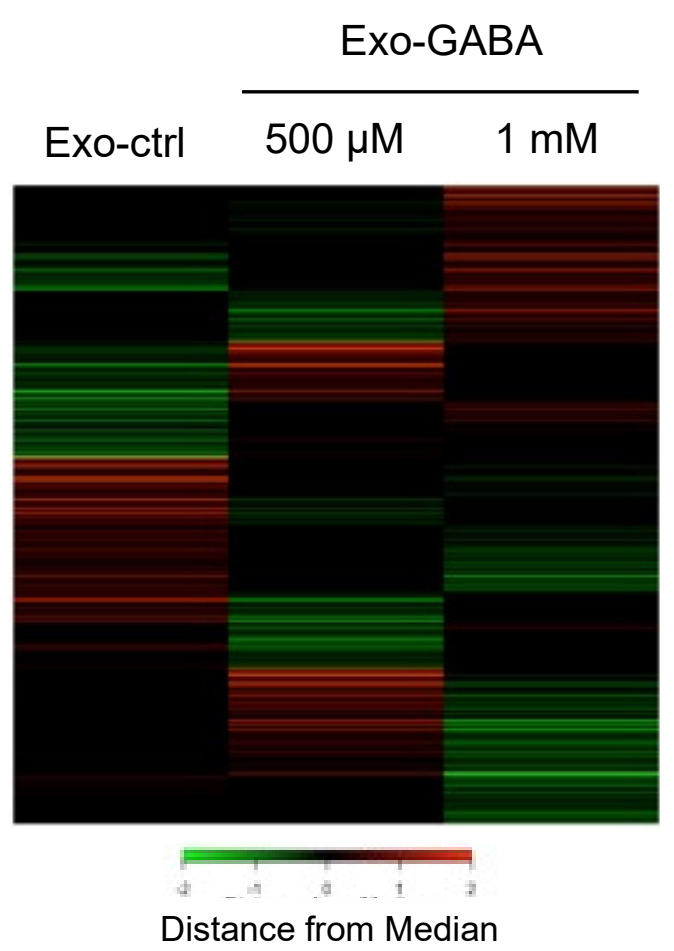

Figure 3. Microarray analysis of genes in exosome-treated SH-SY5Y cells. Heatmap was used to visualize differential expressed genes.

Table 2. Functional classification of genes differentially expressed in SH-SY5Y cells treated with exosomes derived from GABA-treated Caco-2 cells.

\begin{tabular}{ll}
\hline KEGG Pathway & $p$-Value \\
\hline Cytokine-cytokine receptor interaction & 0.0037 \\
Neuroactive ligand-receptor interaction & 0.025 \\
Inflammatory bowel disease & 0.029 \\
\hline
\end{tabular}

We then performed integrated analysis of miRNAs with altered expression in ExoGABA $_{500}$ and Exo-GABA 1000 and of mRNAs with altered expression in SH-SY5Y cells in response to Exo-GABA 500 and Exo-GABA 1000 treatment. After comparison of miRNA target genes whose expression was altered both in Exo-GABA Ex0 $_{00}$ and Exo-GABA 1000 and those whose expression was altered in SH-SY5Y cells in response to Exo-GABA 500 and Exo-GABA $_{1000}, 185$ common genes were found, 12 of which were involved in the regulation of brain function (Table 3 ).

Table 3. Neuronal gene and its function selected by integration analysis.

\begin{tabular}{ll}
\hline Gene & Function \\
\hline CIT & Development of the central nervous system \\
SLC6A17 & Neurotransmitter uptake \\
\hline
\end{tabular}


Table 3. Cont.

\begin{tabular}{ll}
\hline Gene & Function \\
\hline MPPED2 & Brain development \\
NPAS3 & Neurogenesis \\
SHANK2 & Scaffolding at the synapse \\
NEUROG1 & Neuronal differentiation \\
RIT1 & Neuron development and regeneration \\
SLC5A7 & Depression \\
GCSAM & Signaling pathway \\
KCNN2 & Regulation of neuronal excitability \\
KCNK13 & Neurotransmitter release \\
KAT6B & Brain development \\
\hline
\end{tabular}

\subsection{Effects of Exosomes Derived from the Serum of GABA-Treated Mice in SH-SY5Y Cells}

First, we tested whether serum-derived exosomes from mice orally administered GABA (seExo-GABA) could induce neurite outgrowth in SH-SY5Y cells. After isolating exosomes from serum of mice administered GABA, SH-SY5Y cells were similarly treated with seExo-GABA (equivalent to $90 \mathrm{ng}$ protein) for $24 \mathrm{~h}$, as mentioned above. The results clearly showed that seExo-GABA induced neurite outgrowth in SH-SY5Y cells (Figure 4A). Furthermore, seExo-GABA augmented the expression of $P G C-1 \alpha$ and increased the number and area of mitochondria in SH-SY5Y cells, but not activity (Figure 4B-E).

A

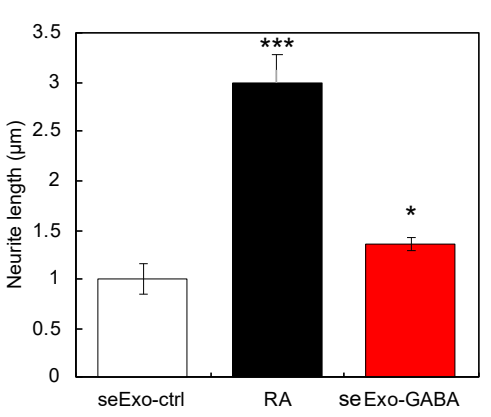

C

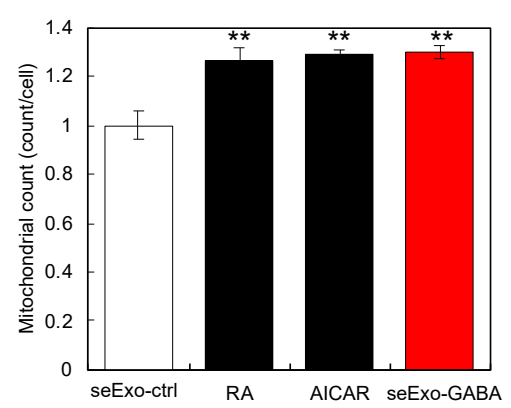

E

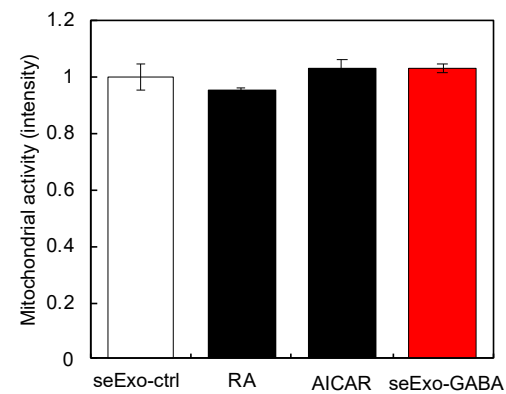

B

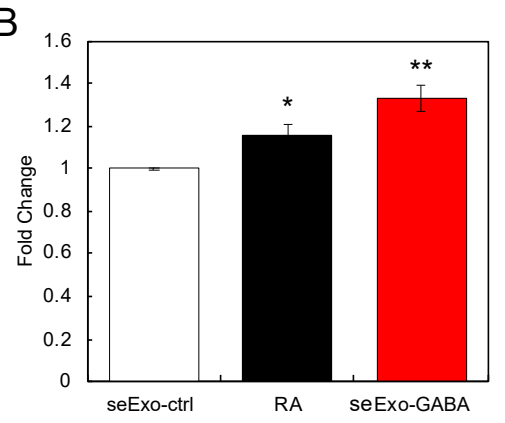

D

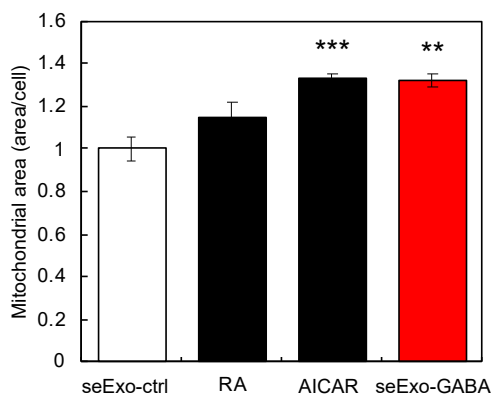

Figure 4. Effects of exosomes derived from serum of GABA-treated mice (seExo-GABA) on SH-SY5Y 
cells. Exosomes derived from serum of non-treated mice (seExo-ctrl) was used as control. Neurite length (A) and PGC-1 $\alpha$ expression (B) in SH-SY5Y cells treated with seExo-GABA. Count (C), area (D), and activity (E) of mitochondria in SH-ST5Y cells treated with seExo-GABA. RA was used as positive control. AICAR is used as positive control for mitochondrial biogenesis. Multiple comparisons between groups were carried out by one-way ANOVA with Tukey's post-hoc test. Statistical difference was evaluated by comparing to seExo-ctrl. Statistical significance was defined as $p<0.05\left(^{*} p<0.05\right.$; ${ }^{* *} p<0.01 ;{ }^{* * *} p<0.001$ ) (value means $\pm \mathrm{SEM}, n=3$ ).

Next, we tested the effects of seExo-GABA on the expression of neuronal cell marker genes in SH-SY5Y cells. The results showed that seExo-GABA augmented the expression of some of marker genes except Nestin (Figure 5A-C). These results indicated that seExoGABA activated SH-SY5Y cells, and revealed that exosomes, which are capable of activating neuronal cells, circulate in the blood of mice orally administered GABA.
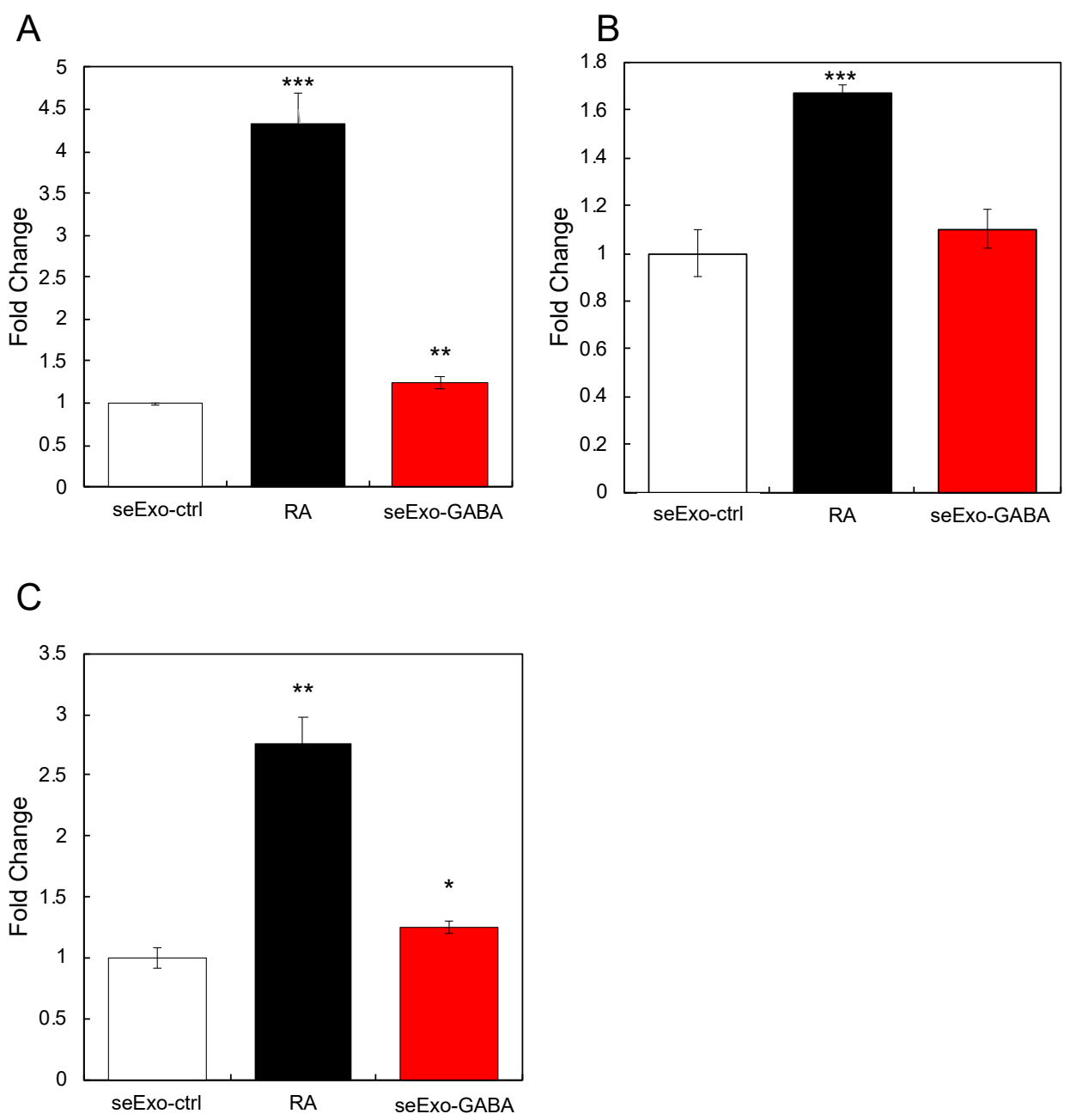

Figure 5. Effects of exosomes derived from serum of GABA-treated mice (seExo-GABA) on gene expression. Exosomes derived from serum of non-treated mice (seExo-ctrl) was used as control. Expression of BDNF (A), Nestin (B), and NEFM (C) in SH-SY5Y cells treated with seExo-GABA was evaluated by RT-qPCR. RA was used as positive control. Multiple comparisons between groups were carried out by one-way ANOVA with Tukey's post-hoc test. Statistical difference was evaluated by comparing to seExo-ctrl. Statistical significance was defined as $p<0.05\left({ }^{*} p<0.05 ;{ }^{* *} p<0.01\right.$; ${ }^{* * *} p<$ 0.001 ) (value means $\pm \mathrm{SEM}, n=3$ ). 


\subsection{Microarray Analysis of Hippocampal Tissue of Mice Orally Administered GABA}

Here, we performed microarray analysis of hippocampal mRNA of mice orally administered GABA. Results showed that in the hippocampus of mice orally administered GABA, 1127 and 249 genes were significantly up- and down-regulated, respectively. Among these genes, many genes were observed to be related to brain function (Tables 4 and 5). Furthermore, GSEA analysis of these genes showed that oral administration of GABA up-regulated the expression of genes related to memory function in the hippocampus (Figure 6).

Table 4. Functional categories of genes differentially expressed in the hippocampus of GABAadministered mice.

\begin{tabular}{cc}
\hline Functional Categories & $p$-Value \\
\hline Synapse & $3.1 \times 10^{-7}$ \\
Zinc-finger & $5.6 \times 10^{-5}$ \\
Postsynaptic membrane & $7.3 \times 10^{-3}$ \\
Actin nucleation & $4.5 \times 10^{-3}$ \\
\hline
\end{tabular}

Table 5. KEGG pathway of genes differentially expressed in hippocampus of GABA-administered mice.

\begin{tabular}{cc}
\hline KEGG Pathway & $p$-Value \\
\hline Long-term potentiation & $2.0 \times 10^{-4}$ \\
Regulation of actin cytoskeleton & $2.2 \times 10^{-4}$ \\
Alzheimer's disease & $5.9 \times 10^{-2}$ \\
Long-term depression & $1.5 \times 10^{-3}$ \\
\hline
\end{tabular}

A

$$
\text { B }
$$
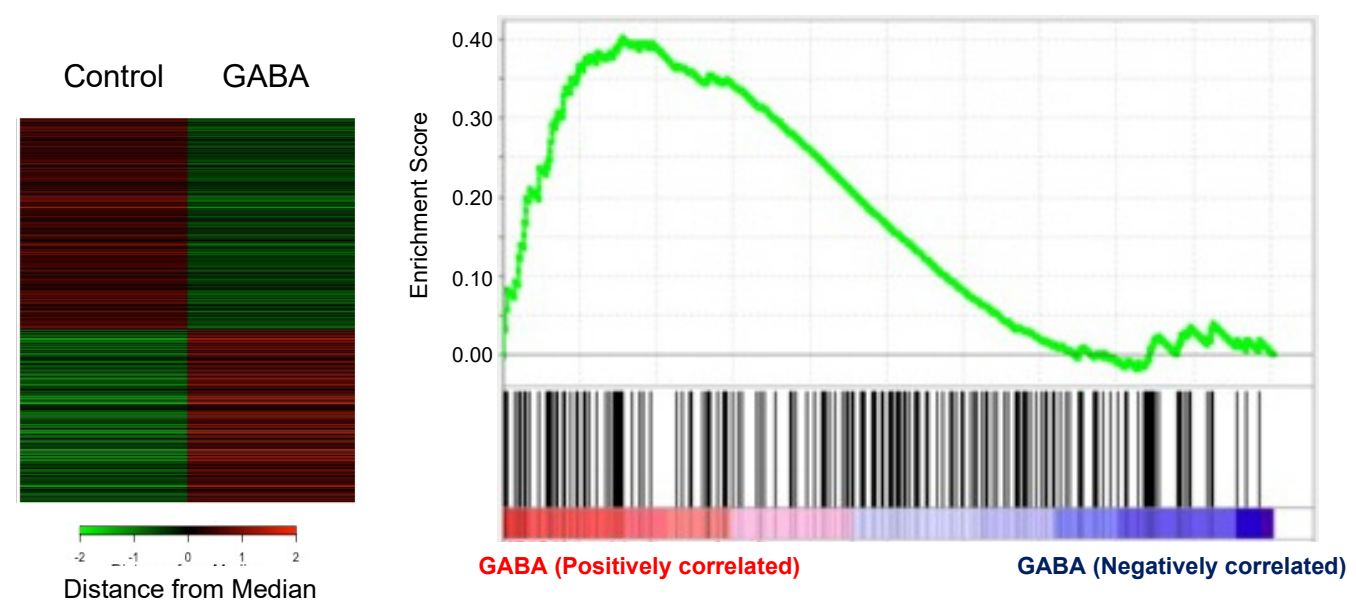

Figure 6. Microarray analysis of genes in the hippocampus of GABA-treated mice. (A) Heatmap was used to visualize differential expressed genes. (B) Gene set enrichment analysis (GSEA) analysis of gene sets relating to memory in the hippocampus of GABA-treated mice. Enrichment plots (green curve) show the running sum of enrichment score (ES) for memory-related gene set. The score at the peak of the plot is the ES for the gene set. The black bars show where the member of the gene set appear in the ranked list of genes. Each black bar represents a memory-related gene. A predominance of black bars to the left or right side indicates that these genes are up-regulated or down-regulated in the hippocampus of GABA-treated mice.

\section{Discussion}

Exosomes derived from various types of cells have been reported to induce neuroprotection and neural recovery by modulating the expression of genes, proteins, and 
miRs in target cells and tissues [31,32]. Emerging evidence shows that exosome-mediated multiple communication axes between various organs such as the brain, heart, kidneys, and intestines, as well as systemic immune responses, can influence health status. In the present study, we showed that GABA activated intestinal cells to secrete exosomes that activate neurons, which may shed light on the exosome-mediated activation of gut-brain interactions caused by GABA. Since it has been reported that $\mathrm{GABA}_{\mathrm{A}}$ receptor is expressed in Caco-2 cells [33], it will be necessary to verify whether GABA activates Caco-2 cells via its receptors to change the content of exosomes, or whether GABA itself is taken up by cells and incorporated into exosomes to function in the target tissue. Recent research has shown that GABA, a type of amino acid, is found in many fermented foods such as yogurt and pickled vegetables. We have previously shown that carnosine, as well as GABA, activates gut-brain interactions via exosomes, but this is a rare example of exosomemediated activation of gut-brain interactions by a food component [24,34]. In this study, we clarified that serum exosomes of mice administered GABA (seExo-GABA) as well as exosomes derived from GABA-treated Caco-2 cells (Exo-GABA) activated neuronal cells to induce neurite growth and mitochondrial activation. These results suggest that these exosomes function as mediators that carry signals to activate neurons, and that certain foods including carnosine and GABA can produce these neuron-activating exosomes. This study could lead to the creation of a new research area: the development of foods that control brain function through the secretion of functional exosomes from the gut.

Microarray analysis of miRNA in Exo-GABA and of mRNA in SH-SY5Y cells treated with Exo-GABA identified signaling pathways which might be activated by Exo-GABA and be involved in GABA-induced phenotypes. Integrated analysis of miRNAs with altered expression in Exo-GABA and of mRNAs with altered expression in SH-SY5Y cells in response to Exo-GABA treatment identified 12 genes which were involved in the GABAinduced activation of brain function. In the future study, we would like to clarify the functionality of these 12 genes in the regulation of brain function.

There are many possible mechanisms by which exosomes regulate neuronal function. In a previous study, we reported that carnosine augmented the expression of miR-6769-5p in exosomes derived from carnosine-treated Caco-2 cells, which led to repression of target gene (ATXN1) expression, thereby activating neurons [34]. With further analysis, we would like to clarify the molecular mechanism of GABA-mediated activation of neuronal cells through exosomes. In particular, it is necessary to verify whether exosomes contain GABA or other bioactive parts in the future.

The present study also showed that serum exosomes of mice administered GABA activated neuronal cells (Figure 7). This result indicated that exosomes, which can activate neurons, circulate in the blood of mice after GABA administration. Indeed, exosomes derived from mesenchymal stromal cells may be useful for remodeling and functional recovery of neurons after stroke by transferring miR-133b to astrocytes and neurons and regulating gene expression [35]. Recently, various functions of milk exosomes derived from bovine milk have been reported. The milk exosome has been shown to carry specific miRNAs (miR-148a) to various target cells and to exert various functions on them. A characteristic example of milk exosome functionalities include effects against $\alpha$-synuclein pathology in Parkinson's disease and Type 2 diabetes mellitus [36]. In other words, both milk exosomes and GABA-derived exosomes are similar in the sense that they are carried in the bloodstream to the target tissue. These reports indicate that serum exosomes are involved in the regulation of brain function, and suggest that by analyzing serum exosomes after ingestion, the functionality of foodstuffs can be verified. Furthermore, it will be possible to develop diagnostic methods for brain function based on serum exosomes. 


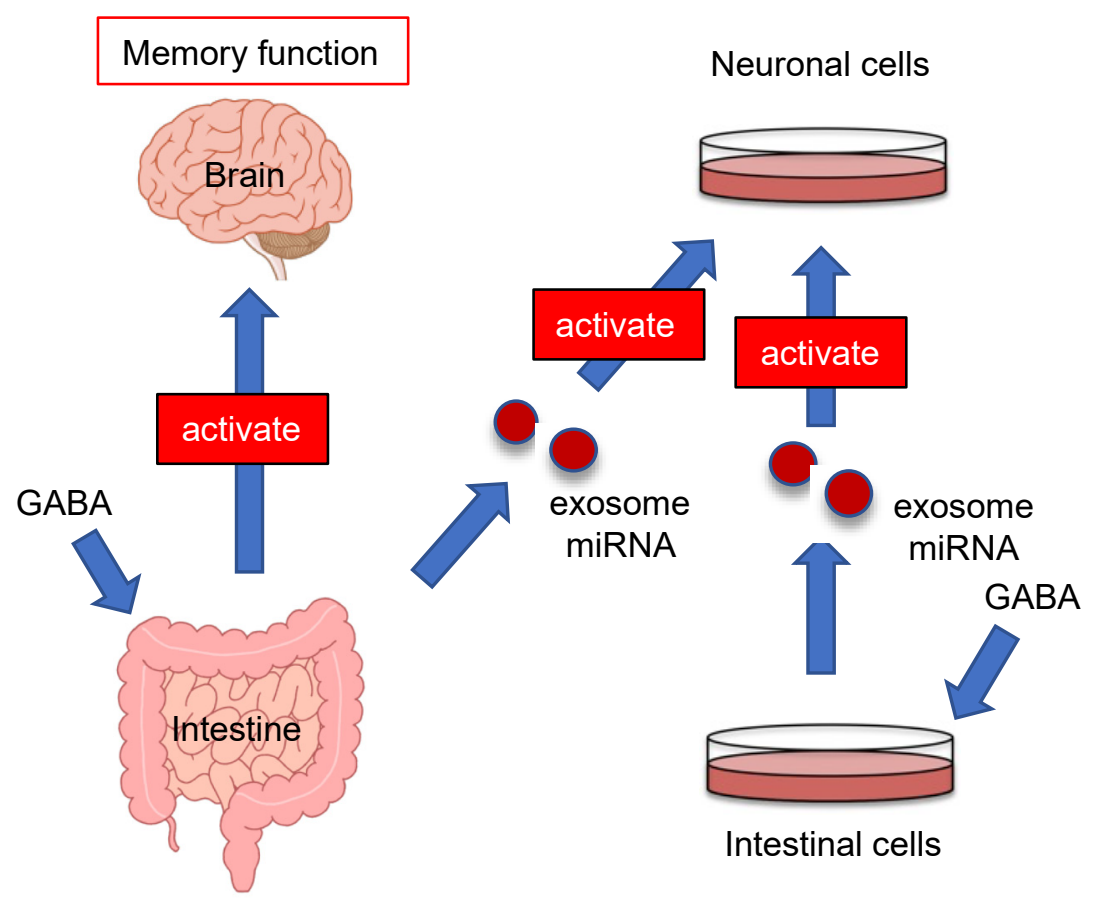

Figure 7. Schematic diagram of GABA function.

To verify the effect of GABA administration on brain function, the effects of GABA on sleep, relaxation, and psychological and physical fatigue have been investigated in humans $[15,18,37]$. These results indicate that GABA strongly affects the early stages of sleep, reduces both psychological and physical fatigue, and acts as a natural relaxant inducer. These results also suggest that GABA may also contribute to improved brain function, especially memory function. GSEA analysis of genes in the hippocampus of GABA-treated mice revealed that GABA administration could have some effect on brain function through the regulation of genes related to memory function. The detailed molecular mechanisms by which GABA regulate gene expression in the hippocampus need to be clarified in the future.

\section{Conclusions}

In this study, we clarified that serum exosomes of mice administered GABA (seExoGABA) as well as exosomes derived from GABA-treated Caco-2 cells (Exo-GABA) activated neuronal cells to induce neurite growth and mitochondrial activation. These results suggest that these exosomes function as mediators that carry signals to activate neurons, and that certain foods including GABA can produce these neuron-activating exosomes. This study could lead to the creation of a new research area: the development of foods that control brain function through the secretion of functional exosomes from the gut.

Supplementary Materials: The following are available online at https://www.mdpi.com/article/10 $.3390 /$ nu13082544/s1, Table S1: Target genes of four miRNA.

Author Contributions: Conceptualization, A.Y., M.K. and Y.K.; methodology, Y.K.; validation, M.U.; investigation, R.I.; data curation, M.U.; writing-original draft preparation, Y.K.; writing-review and editing, Y.K.; project administration, Y.K.; funding acquisition, Y.K. All authors have read and agreed to the published version of the manuscript.

Funding: Y.K. was supported by JSPS KAKENHI (Grant Number 16K14929, 21H02141). Y.K. received the collaborative research cost from Pharma Foods International Co., Ltd.

Institutional Review Board Statement: The study was conducted according to the guidelines of the Declaration of Helsinki, and approved by the Ethics Committee on Animal Experimentation of Kyushu University (approval number: A28-187-0). 
Informed Consent Statement: Not applicable.

Data Availability Statement: The data that support the findings of this study are available from the corresponding author, Y.K., upon reasonable request.

Acknowledgments: The authors would like to thank G. Takada and N. Oshima (GE Healthcare) for their expert assistance with the IN Cell Analyzer 2200, and K. Yasuda (Cell Innovator, Fukuoka, Japan) for her assistance with microarray analysis.

Conflicts of Interest: Authors from Pharma Foods International Co., Ltd. had no role in the design of the study; in the collection, analyses, or interpretation of data; in the writing of the manuscript, or in the decision to publish the results.

\section{References}

1. Obata, K. Synaptic Inhibition and $\gamma$-Aminobutyric Acid in the Mammalian Central Nervous System. Proc. Jpn. Acad. Ser. B Phys. Biol. Sci. 2013, 89, 139-156. [CrossRef] [PubMed]

2. Park, J.; Fu, Z.; Frangaj, A.; Liu, J.; Mosyak, L.; Shen, T.; Slavkovich, V.N.; Ray, K.M.; Taura, J.; Cao, B.; et al. Structure of human GABA B receptor in an inactive state. Nature 2020, 584, 304-309. [CrossRef] [PubMed]

3. Scott, S.; Aricescu, A.R. A structural perspective on GABAA receptor pharmacology. Curr. Opin. Struct. Biol. 2019, 54, 189-198. [CrossRef]

4. Stagg, C.J.; Bachtiar, V.; Johansen-Berg, H. The Role of GABA in Human Motor Learning. Curr. Biol. 2011, 21, 480-484. [CrossRef]

5. Floyer-Lea, A.; Wylezinska, M.; Kincses, T.; Matthews, P.M. Rapid Modulation of GABA Concentration in Human Sensorimotor Cortex During Motor Learning. J. Neurophysiol. 2006, 95, 1639-1644. [CrossRef]

6. Loerch, P.M.; Lu, T.; Dakin, K.A.; Vann, J.M.; Isaacs, A.; Geula, C.; Wang, J.; Pan, Y.; Gabuzda, D.H.; Li, C.; et al. Evolution of the Aging Brain Transcriptome and Synaptic Regulation. PLoS ONE 2008, 3, e3329. [CrossRef] [PubMed]

7. Stanley, D.P.; Shetty, A.K. Aging in the Rat Hippocampus Is Associated With Widespread Reductions in the Number of Glutamate decarboxylase-67 Positive Interneurons but Not Interneuron Degeneration. J. Neurochem. 2004, 89, 204-216. [CrossRef] [PubMed]

8. Robinson, L.C.; Barat, O.; Mellott, J.G. GABAergic and glutamatergic cells in the inferior colliculus dynamically express the $\mathrm{GABA}_{\mathrm{A}} \mathrm{R} \gamma_{1}$ subunit during aging. Neurobiol. Aging 2019, 80, 99-110. [CrossRef]

9. Hua, T.; Kao, C.; Sun, Q.; Li, X.; Zhou, Y. Decreased Proportion of GABA Neurons Accompanies Age-Related Degradation of Neuronal Function in Cat Striate Cortex. Brain Res. Bull. 2008, 75, 119-125. [CrossRef] [PubMed]

10. Han, S.; Tai, C.; Westenbroek, R.E.; Yu, F.H.; Cheah, C.S.; Potter, G.B.; Rubenstein, J.L.; Scheuer, T.; de la Iglesia, H.O.; Catterall, W.A. Autistic-Like Behaviour in Scn1a+/ - Mice and Rescue by Enhanced GABA-Mediated Neurotransmission. Nature 2012, 489, 385-390. [CrossRef]

11. Verret, L.; Mann, E.O.; Hang, G.B.; Barth, A.M.I.; Cobos, I.; Ho, K.; Devidze, N.; Masliah, E.; Kreitzer, A.C.; Mody, I.; et al. Inhibitory Interneuron Deficit Links Altered Network Activity and Cognitive Dysfunction in Alzheimer Model. Cell 2012, 149, 708-721. [CrossRef]

12. Xu, Y.; Zhao, M.; Han, Y.; Zhang, H. GABAergic Inhibitory Interneuron Deficits in Alzheimer's Disease: Implications for Treatment. Front. Neurosci. 2020, 14, 660. [CrossRef] [PubMed]

13. Hosinian, M.; Qujeq, D.; Ahangar, A.A. The Relation Between GABA and L-Arginine Levels With Some Stroke Risk Factors in Acute Ischemic Stroke Patients. Int. J. Mol. Cell. Med. 2016, 5, 100-105.

14. Yamatsu, A.; Nakamura, U.; Saddam, H.M.; Horie, N.; Kaneko, T.; Kim, M. Improvement of Memory and Spatial Cognitive Function by Continuous Ingestion of $100 \mathrm{mg} /$ Day of $\gamma$-Aminobutyric Acid (GABA)-A Randomized, Double-Blind, PlaceboControlled Parallel-Group Clinical Trial-. Jpn. Pharmacol. Ther. 2020, 48, 475-486.

15. Abdou, A.M.; Higashiguchi, S.; Horie, K.; Kim, M.; Hatta, H.; Yokogoshi, H. Relaxation and Immunity Enhancement Effects of $\gamma$-Aminobutyric Acid (GABA) Administration in Humans. BioFactors 2006, 26, 201-208. [CrossRef] [PubMed]

16. Nakamura, H.; Takishima, T.; Kometani, T.; Yokogoshi, H. Psychological Stress-Reducing Effect of Chocolate Enriched With $\gamma$-Aminobutyric Acid (GABA) in Humans: Assessment of Stress Using Heart Rate Variability and Salivary Chromogranin A. Int. J. Food Sci. Nutr. 2009, 60 (Suppl. 5), 106-113. [CrossRef] [PubMed]

17. Yamatsu, A.; Yamashita, Y.; Maru, I.; Yang, J.; Tatsuzaki, J.; Kim, M. The Improvement of Sleep by Oral Intake of GABA and Apocynum venetum Leaf Extract. J. Nutr. Sci. Vitaminol. (Tokyo) 2015, 61, 182-187. [CrossRef]

18. Kanehira, T.; Nakamura, Y.; Nakamura, K.; Horie, K.; Horie, N.; Furugori, K.; Sauchi, Y.; Yokogoshi, H. Relieving Occupational Fatigue by Consumption of a Beverage Containing $\gamma$-Amino Butyric Acid. J. Nutr. Sci. Vitaminol. (Tokyo) 2011, 57, 9-15. [CrossRef]

19. Boonstra, E.; de Klejin, R.; Colzato, L.S.; Alkemade, A.; Forstmann, B.U.; Nieuwenhuis, S. Neurotransmitters as food supplements: The effects of GABA on brain and behavior. Front. Psychol. 2015, 6, 1520. [CrossRef]

20. Cataldo, P.G.; Villena, J.; Elean, M.; de Giori, G.S.; Saavedra, L.; Hebert, E.M. Immunomodulatory Properties of a $\gamma$-Aminobutyric Acid-Enriched Strawberry Juice Produced by Levilactobacillus brevis CRL 2013. Front. Microbiol. 2020, 11, 610016. [CrossRef]

21. Al-Kuraishy, H.M.; Hussian, N.R.; Al-Naimi, M.S.; Al-Gareeb, A.I.; Al-Mamorri, F.; Al-Buhadily, A.K. The Potential Role of Pancreatic $\gamma$-Aminobutyric Acid (GABA) in Diabetes Mellitus: A Critical Reappraisal. Int. J. Prev. Med. 2021, 12, 19. 
22. An, J.; Seok, H.; Ha, E.M. GABA-producing Lactobacillus plantarum inhibits metastatic properties and induces apoptosis of 5-FU-resistant colorectal cancer cells via GABAB receptor signaling. J. Microbiol. 2021, 59, 202-216. [CrossRef]

23. Zareian, M.; Oskoueian, E.; Majdinasab, M.; Forghani, B. Production of GABA-enriched idli with ACE inhibitory and antioxidant properties using Aspergillus oryzae: The antihypertensive effects in spontaneously hypertensive rats. Food Funct. 2020, 11, 4304-4313. [CrossRef]

24. Inotsuka, R.; Uchimura, K.; Yamatsu, A.; Kim, M.; Katakura, Y. $\gamma$-Aminobutyric Acid (GABA) Activates Neuronal Cells by Inducing the Secretion of Exosomes From Intestinal Cells. Food Funct. 2020, 11, 9285-9290. [CrossRef]

25. Bazzan, E.; Tinè, M.; Casara, A.; Biondini, D.; Semenzato, U.; Cocconceli, E.; Baestro, E.; Damin, M.; Radu, C.M.; Taruto, G.; et al. Critical Review of the Evolution of Extracellular Vesicles' Knowledge: From 1946 to Today. Int. J. Mol. Sci. 2021, $22,6417$. [CrossRef]

26. Livak, K.J.; Schmittgen, T.D. Analysis of relative gene expression data using real-time quantitative PCR and the $2^{-\Delta \Delta C T}$ method. Methods 2001, 25, 402-408. [CrossRef] [PubMed]

27. Bolstad, B.M.; Irizarry, R.A.; Åstrand, M.; Speed, T.P. A Comparison of Normalization Methods for High Density Oligonucleotide Array Data Based on Variance and Bias. Bioinformatics 2003, 19, 185-193. [CrossRef] [PubMed]

28. Huang, D.W.; Sherman, B.T.; Lempicki, R.A. Bioinformatics Enrichment Tools: Paths Toward the Comprehensive Functional Analysis of Large Gene Lists. Nucl. Acids Res. 2009, 37, 1-13. [CrossRef] [PubMed]

29. Huang, D.W.; Sherman, B.T.; Lempicki, R.A. Systematic and Integrative Analysis of Large Gene Lists Using DAVID Bioinformatics Resources. Nat. Protoc. 2009, 4, 44-57. [CrossRef]

30. Fu, X.; Wan, S.; Lyu, Y.L.; Liu, L.F.; Qi, H. Etoposide induces ATM-dependent mitochondrial biogenesis through AMPK activation. PLOS ONE 2008, 6, e2009. [CrossRef]

31. Venkat, P.; Chen, J.; Chopp, M. Exosome-Mediated Amplification of Endogenous Brain Repair Mechanisms and Brain and Systemic Organ Interaction in Modulating Neurological Outcome After Stroke. J. Cereb. Blood Flow Metab. 2018, 38, $2165-2178$. [CrossRef]

32. Xin, H.; Li, Y.; Chopp, M. Exosomes/miRNAs as Mediating Cell-Based Therapy of Stroke. Front. Cell. Neurosci. $2014,8,377$. [CrossRef] [PubMed]

33. Ma, X.; Sun, Q.; Sun, X.; Chen, D.; Wei, C.; Yu, X.; Liu, C.; Li, Y.; Li, J. Activation of GABA receptors in colon epithelium exacerbates acute colitis. Front. Immunol. 2018, 9, 987. [CrossRef] [PubMed]

34. Sugihara, Y.; Onoue, S.; Tashiro, K.; Sato, M.; Hasegawa, T.; Katakura, Y. Carnosine Induces Intestinal Cells to Secrete Exosomes That Activate Neuronal Cells. PLoS ONE 2019, 14, e0217394. [CrossRef]

35. Xin, H.; Li, Y.; Liu, Z.; Wang, X.; Shang, X.; Cui, Y.; Zhang, Z.G.; Chopp, M. MiR-133b Promotes Neural Plasticity and Functional Recovery After Treatment of Stroke With Multipotent Mesenchymal Stromal Cells in Rats via Transfer of Exosome-Enriched Extracellular Particles. Stem Cells 2013, 31, 2737-2746. [CrossRef]

36. Melnik, B.C. Synergistic Effects of Milk-Derived Exosomes and Galactose on $\alpha$-Synuclein Pathology in Parkinson's Disease and Type 2 Diabetes Mellitus. Int. J. Mol. Sci. 2021, 22, 1059. [CrossRef] [PubMed]

37. Yamatsu, A.; Yamashita, Y.; Pandharipande, T.; Maru, I.; Kim, M. Effect of Oral $\gamma$-Aminobutyric Acid (GABA) Administration on Sleep and Its Absorption in Humans. Food Sci. Biotechnol. 2016, 25, 547-551. [CrossRef] 